\title{
Assessment of soluble angiogenic markers in pancreatic cancer
}

\author{
Cristiana Pistol-Tana $e^{1 \dagger}$, \\ Elena Raducan ${ }^{1}$, \\ Simona O limpia D ima², \\ Lucian Albulescu ${ }^{1}$, \\ Ionita Alina ${ }^{1}$, \\ Poroschianu M arius ${ }^{1}$, \\ Linda Maria Cruceru ${ }^{3}$, \\ Eleonora Codorean ${ }^{1}$, \\ Teodora M onica N eagu ${ }^{1}$ \\ \& Irinel Popescu ${ }^{2}$ \\ ${ }^{\dagger}$ Author for correspondence \\ ${ }^{1}$ 'Victor Babes' National \\ Institute of Pathology, \\ 99-101 Splaiul \\ Independentei, Bucharest, \\ Romania \\ Tel.: +40 213194528 ; \\ Fax: +40 213194 528; \\ E-mail: bioch@vbabesro \\ ${ }^{2}$ Fundeni Clinical Institute, \\ 258 Soseaua Fundeni, \\ Bucharest, Romania \\ Tel.: +40 788015 976 \\ E-mail: dima.simona@ \\ gmail.com \\ ${ }^{3}$ D epartment of C ellular \& \\ $M$ olecular M edicine, \\ 'Carol Davila' University \\ of M edicine \& Pharmacy, \\ 8 Eroilor Sanitari Boulevard, \\ Bucharest, Romania \\ Tel.: +40 723169 349; \\ E-mail: maria_lindabv@ \\ yahoo.com
}

Keywords: angiogenesis, bFGF, Ki67, pancreatic cancer, soluble angiogenic biomarkers, VEGF

fitured excentisg

\begin{abstract}
Angiogenic markers such as VEGF/basic FGF (bFGF) can enlarge the diagnostic biomarkers panel for pancreatic cancer. Materials \& methods: Serum samples from 32 stage I-IV pancreatic cancer patients and 20 controls were analyzed for soluble VEGF/bFGF by ELISA and XMAP array. Results: VEGF/bFGF serum levels were significantly increased in patients compared with controls $(p<0.0001)$. We report a correlation with tumor diameter $(p<0.01 / p<0.05)$, stage $(p<0.001)$, Ki67LI $(p<0.005 / p<0.05)$ and carbohydrate 19-9 antigen ( $p<0.005 / p<0.001$ ). VEGF/bFGF levels analyzed by XM AP array were comparable with the pattern (patient/control) outline obtained by ELISA tests. We obtained a good correlation between these two soluble angiogenic markers $(p<0.001)$. Conclusion: Data obtained for angiogenic markers qualifies them as important candidates in the pancreatic cancer biomarker panel.
\end{abstract}

Exocrine pancreatic carcinoma is now the fifth leading cause of cancer in the USA, Japan and Europe, with an overall 5-year survival rate of less than $5 \%$. O ne of the major causes of death is peritoneal dissemination and liver metastasis [1]. Although pancreatic cancer is not a highly vascularized tumor, its growth depends on the development of an adequate blood supply through angiogenesis at both primary and secondary sites. The pivotal role of angiogenesis in primary tumor growth and metastasis has been recognized for many years [2,3]. Activation of the angiogenic switch during early stages of tumor development suggests that regulation of the angiogenic process is likely to be a ratelimiting step in the progression from small lesion to extensive disease. Several growth factors and their specific receptors have been detected at the tumor site: the VEGF family, FGF family, PDGFs and TGFs. The VEGF family is an important group of signaling proteins involved in pathological angiogenesis. The VEGF family is secreted by tumor cells in the majority of cancers and acts on endothelial cells, promoting new blood-vessel formation. VEGF expression is driven by many factors that are characteristic of tumors, including oncogene expression and hypoxia. Moreover, VEGF mRNA expression can be induced by some of the principal proangiogenic regulators that include growth factors (e.g., FGF, PDGF, TGF and EGF) and cytokines [4-7]. The binding of VEG F to its specific receptor activates intracellular signaling pathways leading to cell proliferation, migration, survival, sprouting and tube formation; upregulation of the molecules involved in degradation of the extracellular matrix further allows proliferating cells to migrate [7].

The FGF family exerts several biological effects, including tumor growth and angiogenesis [8]. These factors stimulate the proliferation of fibroblasts and endothelial cells that give rise to angiogenesis. Basic FGF (bFGF), known as FGF-2, is highly involved in the promotion of endothelial cell proliferation and the physical organization of endothelial cells into tube-like structures, thus promoting angiogenesis [3]. bFGF, along with VEGF or PDGF, is a potent angiogenic factor.

The extent of angiogenesis depends on the balance between proangiogenic and antiangiogenic factors released by tumor and host cell [9]. The expression of VEGF, at present regarded as the major proangiogenic factor for most types of human cancers, is strongly induced by EG Fs and TGFs. H uman pancreatic cancer cells secrete the proangiogenic molecules VEG F and bFGF [10]. It is well established that human pancreatic cancer tissue and cell lines overexpress VEG F [2,11-13].

There is no effective early screening test in pancreatic cancer, and symptoms only appear during late-stage disease when the tumor has metastasized and invaded surrounding tissues. Aiming to enlarge the panel of possible markers detecting the evolution of pancreatic cancer, we have studied soluble VEGFs and bFG Fs in sera harvested from patients diagnosed with stage I-IV pancreatic cancer. We have focused on the correlation between soluble VEGF, bFGF and the disease stage in relation to conventional 


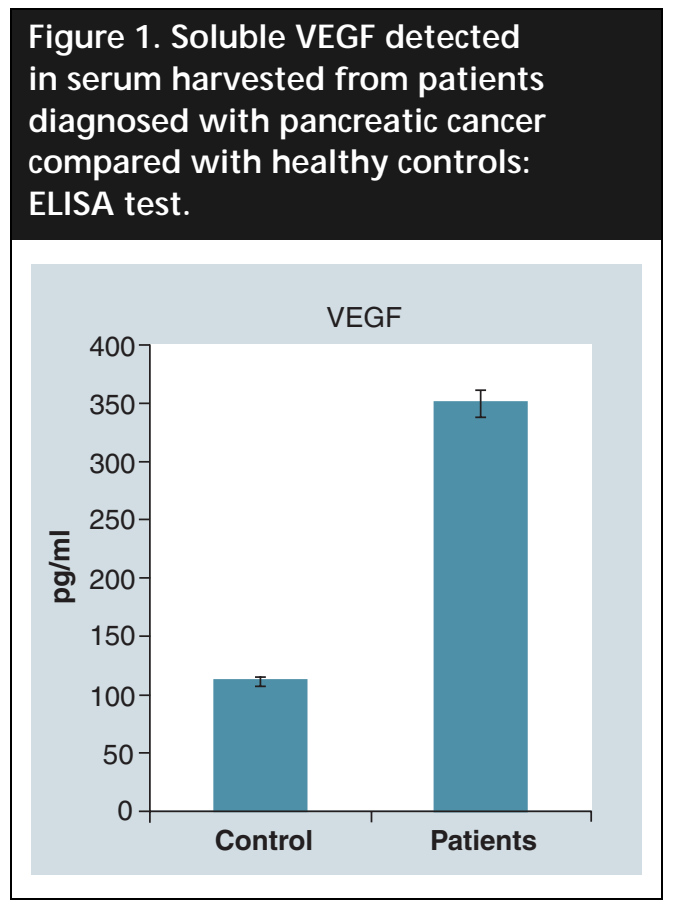

markers. The previously mentioned angiogenic factors were quantified using both ELISA and XM AP technology for simultaneous detection of parameters.

\section{Materials \& methods}

Subjects

The study included 32 patients with pancreatic cancer diagnosed in the Surgical D epartment of Fundeni Clinical Institute (2003-2007); mean age was 55 years (range: $32-74$ years); and 22 men and ten women took part. Tumor staging was based on radiological reports, operative findings and pathology reports in accordance with the T N M system. N one of the patients had undergone any treatment when tested.

The control group consisted of 20 healthy volunteers (12 men and eight women; mean age: 57 years; range: $25-70$ years). The absence of disease was confirmed by physical examination, clinical history and routine laboratory tests, including liver and renal function tests. All patients and heal thy controls provided informed, written consent and the study was approved by the Ethics Committee of Fundeni Clinical Institute and the Victor Babes $\mathrm{N}$ ational Institute of Pathology.

\section{Sample collection}

Blood samples were collected into serum-separating tubes (Vacutainer Systems, Becton Dickinson). Theblood was allowed to clot for $30 \mathrm{~min}$ before centrifugation for $10 \mathrm{~min}$ at $1000 \mathrm{~g}$. Serum was removed and stored in aliquot at $-80^{\circ} \mathrm{C}$ until assayed.

\section{VEGF \& bFGF: ELISA}

Serum levels of angiogenic markers VEGF and bFGF were measured by the immunoenzymatic ELISA method (Quantikine Human VEGF Immunoassay and Quantikine Human FGF Basic Immunoassay, $R \& D$ Systems, $M$ inneapolis, MS, USA) according to manufacturer

\begin{tabular}{|c|c|c|c|c|c|c|c|c|c|}
\hline & & \multicolumn{4}{|c|}{ VEGF } & \multicolumn{4}{|c|}{ bFGF } \\
\hline & & $<300$ & $\geq 300$ & $x^{2}$ & p value & $<25$ & $\geq 25$ & $x^{2}$ & p value \\
\hline \multirow[t]{2}{*}{ Gender } & M & 11 & 11 & 0.27 & $p=0.6$ & 11 & 11 & 0.27 & $p=0.6$ \\
\hline & $\mathrm{F}$ & 4 & 6 & & & 4 & 6 & & \\
\hline \multirow[t]{2}{*}{ Age } & $<60$ & 10 & 13 & 0.37 & $p=0.54$ & 9 & 14 & 1.96 & $p=0.16$ \\
\hline & $\geq 60$ & 5 & 4 & & & 6 & 3 & & \\
\hline \multirow[t]{2}{*}{ Diameter } & $<3$ & 13 & 7 & 7.03 & $p<0.01$ & 12 & 8 & 3.69 & $p<0.05$ \\
\hline & $\geq 3$ & 2 & 10 & & & 3 & 9 & & \\
\hline \multirow[t]{2}{*}{ Stage } & $1+2$ & 15 & 6 & 14.78 & $p<0.001$ & 14 & 7 & 9.61 & $p<0.001$ \\
\hline & $3+4$ & 0 & 11 & & & 1 & 10 & & \\
\hline \multirow[t]{2}{*}{ Ki67 LI } & $<20$ & 9 & 2 & 8.21 & $p<0.005$ & 8 & 3 & 4.5 & $p<0.05$ \\
\hline & $\geq 20$ & 6 & 15 & & & 7 & 14 & & \\
\hline \multirow[t]{2}{*}{ CA 19-9 } & $<40$ & 8 & 1 & 8.87 & $p<0.005$ & 9 & 0 & 14.2 & $p<0.001$ \\
\hline & $\geq 40$ & 7 & 16 & & & 6 & 17 & & \\
\hline \multirow[t]{2}{*}{ CEA } & $<2.5$ & 12 & 8 & 5.72 & $p<0.01$ & 13 & 7 & 7.03 & $p<0.01$ \\
\hline & $\geq 2.5$ & 2 & 10 & & & 2 & 10 & & \\
\hline
\end{tabular}


Figure 2. Soluble VEGF detected in correlation with the stage of pancreatic cancer.

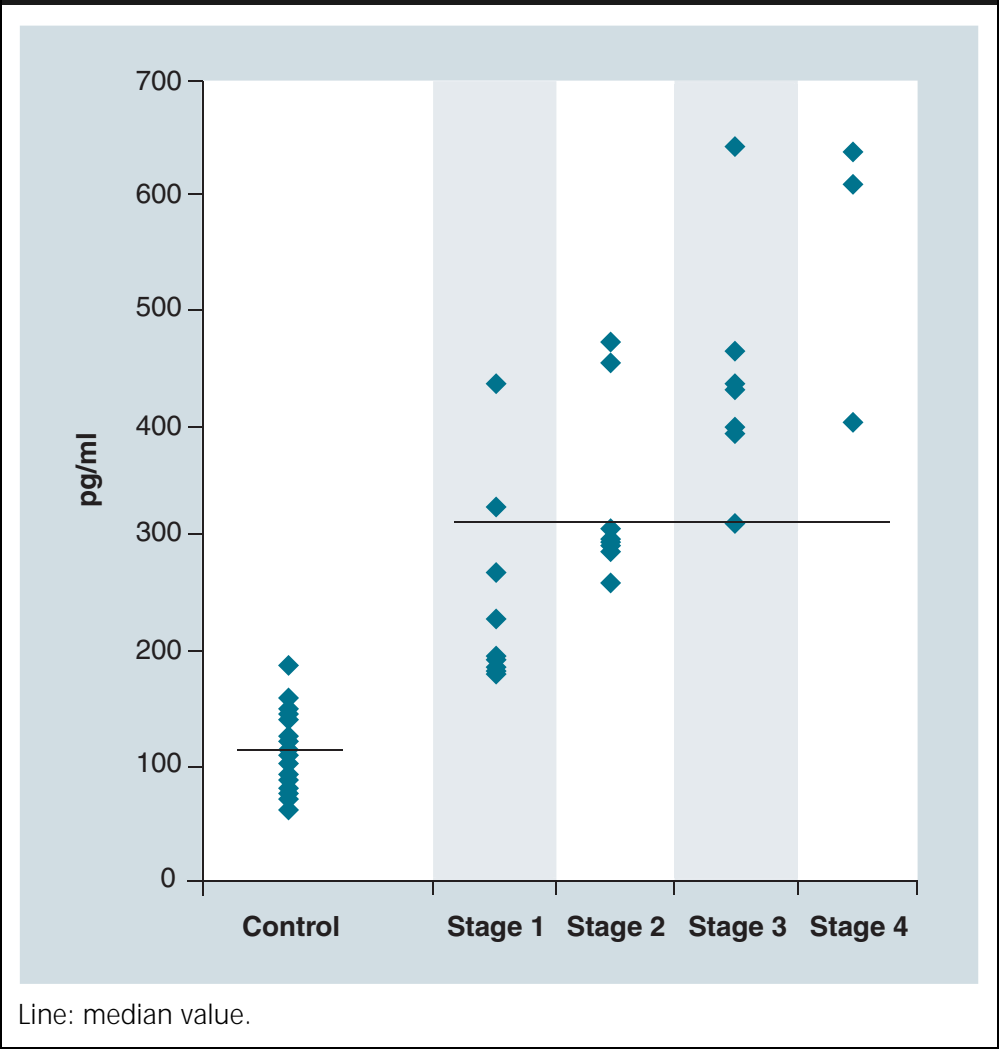

protocol. The absorbance at $450 \mathrm{~nm}$ was measured and concentrations were determined by interpolation of a standard calibration curve. As stated by the supplier, the lower limit of detection is $9 \mathrm{pg} / \mathrm{ml}$ for VEGF and $0.22 \mathrm{pg} / \mathrm{ml}$ for bFGF. All samples were assayed in duplicate and the mean value of $V E G F / b F G F$ was taken into account.

Carcinoembryonic antigen \& carbohydrate 19-9 antigen: ELFA

The serum concentrations for carcinoembryonic antigen (CEA) and carbohydrate 19-9 antigen (CA 19-9) were measured by enzyme-linked fluorescent immunoassay technique ([ELFA], bioM érieux, $M$ arcy l'Etoile, France). The intensity of the fluorescence is proportional to the concentration of CEA and CA 19-9 present in the sample. Test values above $2.5 \mathrm{UI} / \mathrm{ml}$ for CEA and above $37 \mathrm{UI} / \mathrm{ml}$ for CA 19-9 were considered as positive.

VEGF \& bFGF: Luminex XMAP technology In order to extend to high-throughput testing, in addition we have used Luminex $X M A P^{\circledR}$ technology (Luminex ${ }^{\circledR} 200^{m}$, Austin, TX, USA).
The XM AP assay for VEGF and bFGF was performed according to the protocol of $R \& D$ Fluorokine M AP H uman. Plates were analyzed by the Luminex 200 array system.

Statistical analysis

$D$ ata were expressed as the mean \pm standard error of the mean; minimum and maximum values were provided when necessary. The Komolgorov-Smirnov test was used to assess the distribution of data. $D$ ifferences between groups were analyzed by unpaired Student's t-test. p-values less than 0.05 were considered to be statistically significant. The chi-square test $\left(x^{2}, p\right)$ and Pearson correlation $(r, p)$ were used to explore the association between angiogenic marker expression and tumoral markers or clinical parameters. Statistical analysis was performed using SPSS 16.0 software (SPSS Inc., Chicago, IL, USA).

\section{Results}

VEGF levels in pancreatic cancer: correlation with disease stage \& conventional tumor markers

No matter the disease stage, we found a mean level of $349.28 \mathrm{pg} / \mathrm{ml}$ VEGF (range: 181-637 $\mathrm{pg} / \mathrm{ml}$ ), significantly higher than in controls, namely $115.06 \mathrm{pg} / \mathrm{ml}$ (range: 62.16-189 $\mathrm{pg} / \mathrm{ml} ; \mathrm{p}<0.0001$ ) (Figure 1). Similar high values of soluble VEGF in pancreatic cancer patients were found earlier in a comparable study [14], in which the authors found a mean value of $505 \mathrm{pg} / \mathrm{ml}$ in pancreatic cancer patients compared with the $193 \mathrm{pg} / \mathrm{ml}$ found in the control group. Another group previously reported that they had used a cut-off level of $149.5 \mathrm{pg} / \mathrm{ml}$ VEGF to discriminate pancreatic cancer [15]. $W$ ith slightly overlapping values compared with controls, a group studying the correlation of VEGF with the stage of pancreatic cancer obtained a mean value of $294.8 \mathrm{pg} / \mathrm{ml}$ VEG F [16]. We found that serum levels of VEGF correlated significantly with the disease stage and with the standard proliferative marker, Ki67. As the disease stage increased, we found higher VEG F levels ( $\left.x^{2}=14.78 ; p<0.001\right)$ (Figure 2) correlated with Ki67 labeling index (LI) equal to or greater than $20 \%\left(x^{2}=8.21 ; p<0.005\right)$ (Table 1). A dependence of VEGF level on tumor diameter was found, hence patients with tumors larger than $3 \mathrm{~cm}$ in diameter had high VEGF serum levels $\left(x^{2}=7.03 ; p<0.01\right)$ (Table 1). O ther studies correlating hepatocellular carcinoma tumor diameter with VEGF 


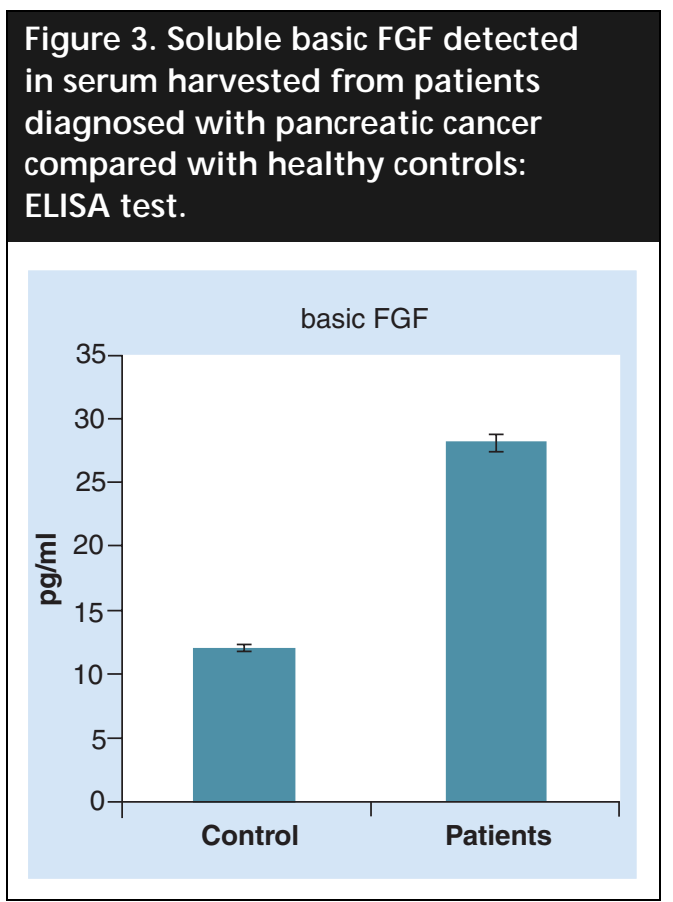

serum values proved that patients bearing tumors over $3 \mathrm{~cm}$ diameter have high soluble VEGF levels [17,18]. We found that when compared with conventional markers, soluble VEGF correlated with an increased level of CA 19-9 $\left(x^{2}=8.87 ; \quad p<0.005\right)$ and CEA $\left(x^{2}=5.72 ; p<0.01\right)$, but no correlation with patient gender or age was recorded (Table 1). In all

\section{Figure 4. Soluble basic FGF detected in correlation $w$ ith the stage of pancreatic cancer.}

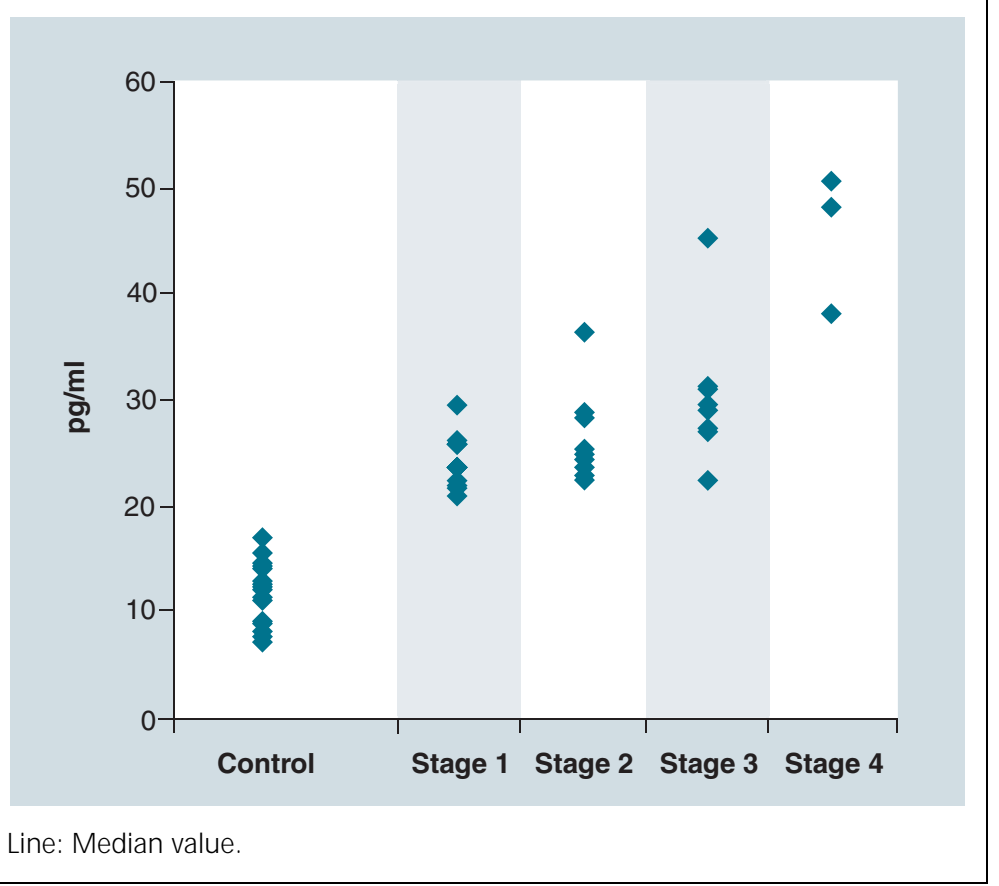

our correlation studies, the threshold employed was $300 \mathrm{pg} / \mathrm{ml} \mathrm{VEGF}$, a value chosen in accordance with our median value and previous reported values.

bFGF levels in pancreatic cancer: correlation with disease stage \& conventional tumor markers

Soluble bF G F in the serum of pancreatic cancer patients had a mean value of $28.19 \mathrm{pg} / \mathrm{ml}$ (range: $20.8-50.6 \mathrm{pg} / \mathrm{ml}$ ), significantly higher compared with control values at $11.76 \mathrm{pg} / \mathrm{ml}$ (range: $7.16-15.4 \mathrm{pg} / \mathrm{ml} ; \quad \mathrm{p}<0.0001$ ) on bFGF (Figure 3). Although there are fewer reports than VEG $F$, there are various soluble bFG $F$ values reported, thus the authors state that $5 \mathrm{pg} / \mathrm{ml}$ soluble bFGF is found in endometrial cancer [19], approximately $15 \mathrm{pg} / \mathrm{ml}$ in colorectal cancer [20] and $60 \mathrm{pg} / \mathrm{ml}$ in B-cell chronic lymphocytic leukemia [21]. The different value obtained in our studies can be attributed to pancreatic cancer being a more aggressive type of neoplasia. We have obtained, as in the case of soluble VEGF, bFGF serum levels significantly correlating with the stage of disease and proliferative marker, Ki67. Following the VEGF pattern, bFGF levels increased in association with the disease stage $\left(x^{2}=9.61\right.$; $\mathrm{p}<0.001$ ) (Figure 4) and with $\mathrm{Ki67} \mathrm{LI}$ equal to or greater than $20 \%\left(x^{2}=4.5 ; p<0.05\right)$. As with VEG F, we found a positive linear correlation with the tumor diameter $\left(x^{2}=3.69\right.$; $p<0.05)$ and with increased levels of classical markers CA 19-9 $\left(x^{2}=14.2 ; p<0.001\right)$ and CEA $\left(x^{2}=7.03 ; p<0.01\right)$. No correlation was found between bFGF levels and the patients' gender or age (Table 1). In current practice, CA 19-9 assays are not optimal for detecting small pancreatic lesions, but correlating this marker with new angiogenic markers can strengthen its power in pancreatic cancer.

In all correlation studies, the threshold used was $25 \mathrm{pg} / \mathrm{ml}$, based on our median value and the reported values in previous studies.

Luminex XM AP technology for angiogenic marker analysis

A subset of serum samples was also analyzed for VEGFs and bFGFs using Luminex XM AP technology. Comparing the control and patient groups, the VEGF/bFGF values obtained by the XMAP array were comparable to the outline obtained by the ELISA tests (Figure 5). T he differences obtained between the control groups and patients were somewhat higher in ELISA tests; 
Figure 5. Soluble VEGF/basic FGF detected by xMAP technology in patients diagnosed with pancreatic cancer compared with controls.

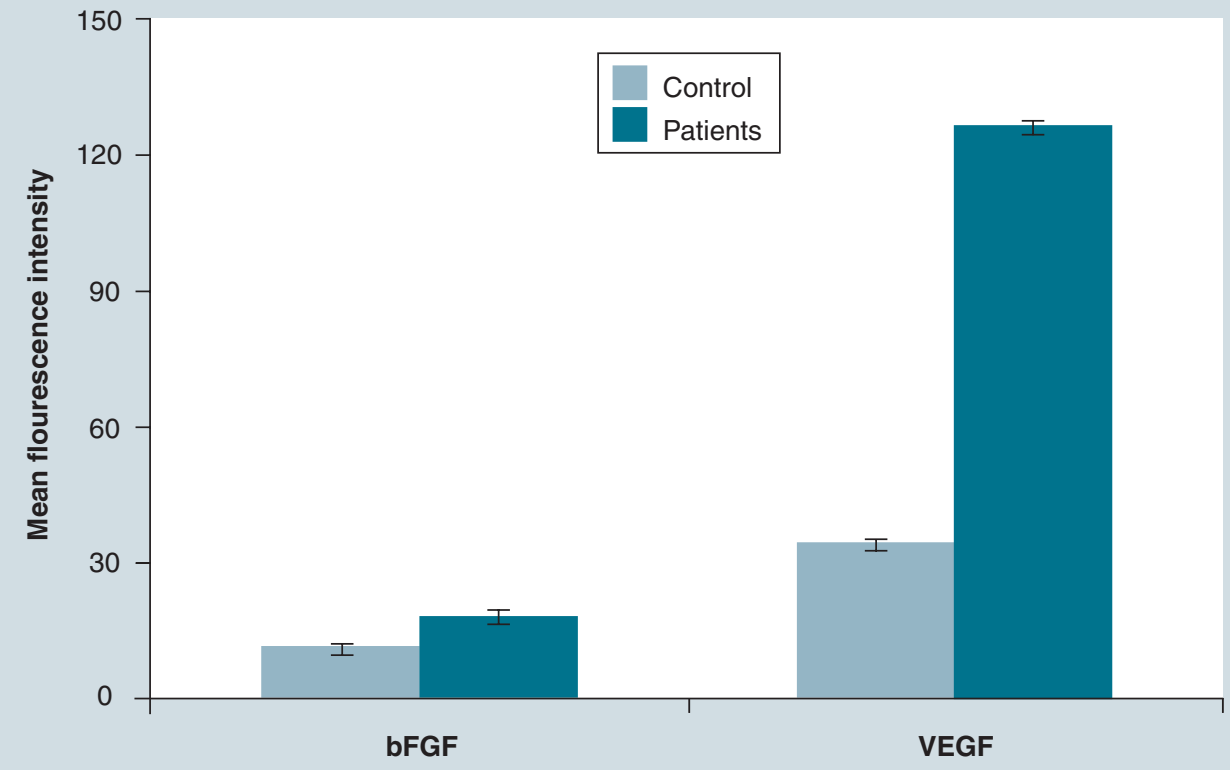

bFGF: Basic FGF.

the mean value for soluble VEGF in patients versus controls was approximately 1.60 -fold greater in Luminex and approximately twofold greater in ELISA analysis. Soluble bFGF mean value was approximately 3.5 -fold in patients versus control when Luminex technology was used, and approximately threefold in ELISA quantification. Comparing the absolute values obtained in both techniques, soluble VEGF detected by XM AP technology was reduced five times compared with ELISA detection, while bFGF was three-times lower.

The high number of samples analyzed, the information gathered concomitantly and the shorter protocol time are obvious advantages in XM AP technology. Nevertheless, interferences may occur, leading to a decrease in the quantified parameters. Another explanation of the differences obtained compared with the ELISA technique is that the monoclonal antibodies used in the kits could have differing specificities [22]. The broader range of values for multiparameter detection (5-10,000 pg/ml) compared with ELISA (VEGF: 0-2000 pg/ml; bFGF: 0-64 pg/ml) can contribute equally to a different detection capacity in samples with high/low marker concentrations.
We have obtained a good correlation between the soluble angiogenic markers VEGF and bFGF $(r=0.7 ; \quad p<0.001 ; \quad$ Pearson correlation) (Figure 6), along with a positive correlation with the classical serum marker CA 19-9 (for VEG F: $r=0.73, p<0.001 ;$ for bFGF: $r=0.70$, $p<0.001$ ) (Table 1). It can be noted that both angiogenic markers follow pancreatic cancer stages from I-IV.

\section{Discussion}

Early diagnosis of pancreatic cancer increases the survival rate of patients, but current clinical pancreatic tumor markers lack the sensitivity and specificity required for early detection. There is still no specific tumor marker for pancreatic cancer; therefore, finding new serum tumor markers of pancreatic cancer has become an important topic in pancreatic surgery [23-26].

The aforementioned simultaneous increase of soluble VEGF and bFGF in pancreatic cancer could be explained by the closely related angiogenic factor pathways sharing common downstream signaling pathways. It seems that the EGFR pathway upregulates VEGF and bFGF, enhancing angiogenesis [10]. The 


\section{Figure 6. Correlation between soluble VEGF and basic FGF in} patients diagnosed with pancreatic cancer.

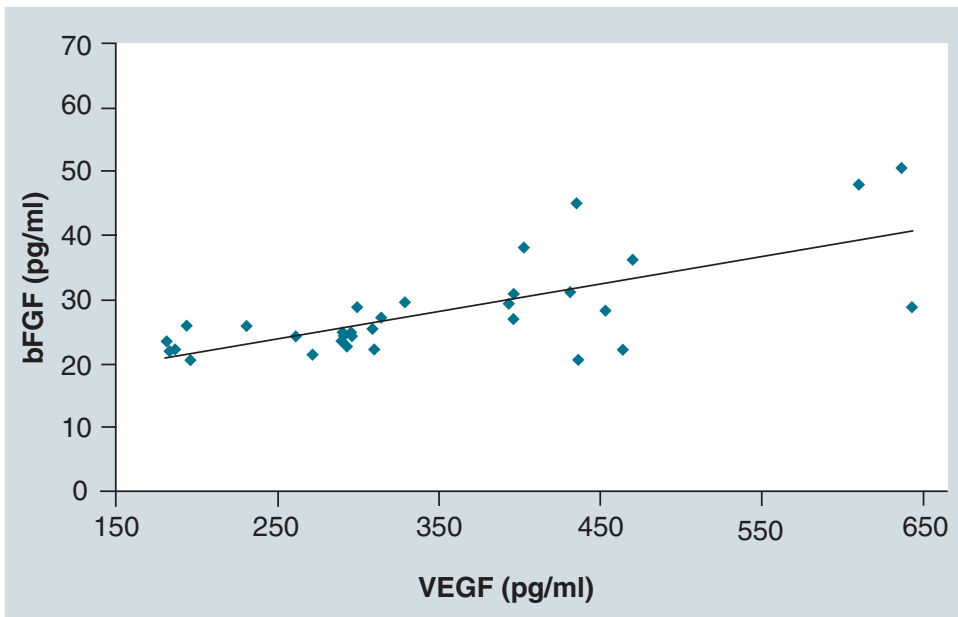

bFGF: Basic FGF.
To our knowledge, this study reports the combined testing of soluble angiogenic markers in pancreatic cancer for the first time. N evertheless, important reports have studied bF GF in combination with VEGF in skin melanomas [31], meningiomas [32], chronic lymphocytic leukemia [33] and nonsmall cell lung cancer [34]. Circulating levels of angiogenic cytokines were reported as having predictive power in neuroendocrine carcinoma of gastro-entero-pancreatic systems [35].

In pancreatic cancer, our results are consistent with other groups that have found significantly higher serum VEGF levels correlated with disease stage and metastases. These findings suggested that serum VEGF concentrations may reflect pancreatic cancer progression, and highlights it as an important marker [14,36]. Later, other groups $[15,16]$ confirmed the high VEG $F$ serum levels in pancreatic cancer patients and pointed it out as a marker to be looked for when antineoplastic agents are used. There is no information regarding soluble bFG $F$ involvement in pancreatic cancer, therefore our study provides data showing a correlation with disease stage, proliferation markers and conventional serum markers. M oreover, we have established a relation between both angiogenic markers and clinicopathological parameters. Furthermore, we detected the angiogenic markers by two systems: the classical ELISA and a newer technology, XM AP. The results obtained displayed a similar pattern regarding controls versus patients.

Comparing ELISA with the XM AP technique, we confirmed that each system has its own merits with regards to screening pancreatic cancer

\section{Table 2. Angiogenic biomarkers: correlation with clinical outcome.}

\begin{tabular}{|c|c|c|c|c|}
\hline Material & VEGF & bFGF & Samples & Ref. \\
\hline Chronic lymphocytic leukemia & $\uparrow$ & $\uparrow$ & Serum & [29] \\
\hline Nonsmall cell lung cancer & $\uparrow$ & $\uparrow$ & Serum & [30] \\
\hline M elanoma, stage III & $\downarrow$ & $\uparrow$ & Serum & [27] \\
\hline Melanoma, stage IV & $\uparrow$ & $\uparrow$ & Serum & [27] \\
\hline Ovarian cancer & $\uparrow$ & - & Serum & [26] \\
\hline Endometrial cancer & - & $\uparrow$ & Serum & [15] \\
\hline Hepatocellular carcinoma & $\uparrow$ & - & Serum & [14] \\
\hline Colorectal cancer & - & $\uparrow$ & Serum & [16] \\
\hline Pancreatic cancer & $\uparrow$ & - & Serum/plasma & [11] \\
\hline Pancreatic cancer & $\uparrow$ & - & Serum & {$[10,12,25,32]$} \\
\hline Pancreatic cancer & $\uparrow$ & $\uparrow$ & Serum & Present results \\
\hline Pancreatic adenocarcinomas & $\uparrow$ & $\uparrow$ & Tissue & [23] \\
\hline Pancreatic ductal carcinoma & $\uparrow$ & $\uparrow$ & Tissue & [24] \\
\hline
\end{tabular}

bFGF: Basic FGF. 
patients, and that different systems display results that cannot be combined [37]. We emphasize that, as recently reported [38], although ELISA and XMAP results are different, the patterns of angiogenic markers generally agree.

Following the development of antiangiogenic drugs, angiogenic-factor testing is useful in order to quantify the response to therapy. Serum VEGF/bFGF levels were reported to be good indicators of antiangiogenic therapy in nonpancreatic cancers $[33,34,39]$.

O ur study focused on the relationship of soluble angiogenic factors, tumor stage and conventional markers. In this respect, soluble VEGFs and bFGFs could be included in the panel of pancreatic cancer biomarkers. To our knowledge, this is the first study correlating the two soluble angiogenic markers in pancreatic cancer. M oreover, we have focused on the high throughput nature of the testing, therefore comparatively using XMAP technology for soluble VEGFs and bFGFs.

\section{Conclusion}

Biomarker discovery for pancreatic cancer is still ongoing, and multiple approaches have been developed for the identification of serum biomarkers. Although angiogenic-soluble factors do not possess the requisite specificity for pancreatic cancer, they can be applied within a panel of protein biomarkers.

VEGFs and bFGFs play important roles in tumor growth and progression. We postulate that blood levels of angiogenic factors may become a tool for the detection of disease recurrence, monitoring of antiangiogenic therapy and selection of patients subjected to VEGF/bFG F-targeted therapies.

In conclusion, this study has demonstrated high soluble VEGF and bFGF levels in pancreatic cancer patients when compared with healthy controls, which correlate with disease stage and tumor progression.

\section{Future perspective}

Early detection of pancreatic adenocarcinoma, diagnosed in its preinvasive state, may greatly impact the treatment and prognosis of patients with fatal malignancy. VEGFs and bFGFs, which probably share common intracellular signaling pathways, and play important roles in tumor growth and progression through the exertion of both indirect and direct effects on tumor cells. Although these angiogenic factors may lack specificity for pancreatic cancer but, in addition to conventional tumor markers, they have the potential to enlarge the panel of protein biomarkers.

The inclusion of angiogenic markers in the pancreatic cancer biomarkers panel could assist in:

\section{- Earlier diagnosis}

- Higher prognosis, recurrence and patient survival rates

- Improved therapy follow-up and individual antiangiogenic therapy

\section{Financial \& competing interests disclosure}

This work was supported by National Research Grant CEEX 64 and PNII 6.1-44. The authors have no other relevant affiliations or financial involvement with any organization or entity with a financial interest in or financial conflict with the subject matter or materials discussed in the manuscript apart from those disclosed.

$\mathrm{No}$ writing assistance was utilized in the production of this manuscript.

\section{Ethical conduct of research}

For investigation involving human subjects, informed consent has been obtained from the participants involved. The authors state that they have obtained appropriate institutional review board approval or have followed the principles outlined in the Declaration of Helsinki for all human or animal experimental investigations. In addition, for investigations invol ving human subjects, informed consent has been obtained from the participants involved.

\section{Executive summary}

- Our findings regarding soluble VEGFs/basic FGFs (bFGFs) in pancreatic cancer suggests that these markers may reflect disease progression, and their quantification could be clinically useful.

- VEGF/bFGF angiogenic markers have a positive correlation with proliferative markers, stage of disease and tumor diameter, and the fact that they relate to conventional markers, such as as carbohydrate 19-9 antigen and carcinoembryonic antigen, makes them important candidates in the pancreatic cancer biomarker panel.

- VEGFs and bFGFs may qualify as markers for prognosis and therapy control in patients with pancreatic cancer. 


\section{Bibliography}

Papers of special note have been highlighted as either of interest $(\bullet)$ or of considerable interest $(\cdot \bullet)$ to readers.

1. Sarkar FH, Banerjee S, Li Y: Pancreatic cancer: pathogenesis, prevention and treatment. Toxicol. Appl. Pharmacol. 224(3), 326-336 (2006).

2. Korc M : Pathways for aberrant angiogenesis in pancreatic cancer. $\mathrm{M} \mathrm{ol}$. Cancer. 7, 2-8 (2003).

-• Summarizes the importance of proangiogenic factors, especially VEG Fs, in pancreatic cancer progression; underlines the correlation between high serum VEG F levels and disease recurrence postoperatively.

3. Tassi $E$, Wellstein A: The angiogenic switch molecule, secreted FGF-binding protein, an indicator of early stages of pancreatic and colorectal adenocarcinoma. Semin. O ncol. 33(6 Suppl. 11), S50-S56 (2006).

4. Summy JM, Trevino JG, Baker $\mathrm{CH}$ et al.: $c$-Src regulates constitutive and $\mathrm{EGF}$ mediated VEG F expression in pancreatic tumor cells through activation of phosphatidyl inositol-3 kinase and p38 M APK. Pancreas 31(3), 263-274 (2005).

5. Cho K, Ishiwata T, U chida $\mathrm{E}$ et al.: Enhanced expression of keratinocyte growth factor and its receptor correlates with venous invasion in pancreatic cancer. Am. J. Pathol. 170(6), 1964-1974 (2007).

6. Ischenko I, Guba M, Yezhelyev M et al.: Effect of Src inhibition on metastasis and tumor angiogenesis in human pancreatic cancer. Angiogenesis 10(3), 167-182 (2007).

7. Tabernero J: The role of VEGF and EGFR inhibition: implications for combining antiVEGF and anti-EGFR agents. $\mathrm{Mol}$. Cancer Res 5(3), 203-220 (2007).

8. M ohammadi M, OIsen SK, Ibrahimi OA et al.: Structural basis for fibroblast growth factor receptor activation. Cytokine G rowth Factor Rev. 16(2), 107-137 (2005).

9. Schuch $\mathrm{G}, \mathrm{Kisker} O$, Atala $\mathrm{A}$ et al.: Pancreatic tumor growth is regulated by the balance between positive and negative modulators of angiogenesis. Angiogenesis 5(3), 181-190 (2002).

- D emonstrates the overexpression of proangiogenic factors VE GF and basic FG F in pancreatic tumor cell cultures, and analyses how changes in the balance between pro- and antiangiogenic factors can regulate tumor growth in vivo.

10. Baker C, Carmen C, Solorzano et al.: Blockade of vascular endothelial growth factor receptor and epidermal growth factor receptor signalling for therapy of metastatic human pancreatic cancer. Cancer Res. 62 , 1996-2003 (2002).

11. Seo Y, Baba H, Fukuda T, Takashima M, Sugimachi $\mathrm{K}$ : $\mathrm{H}$ igh expression of vascular endothelial growth factor is associated with liver metastasis and a poor prognosis for patients with ductal pancreatic adenocarcinoma. Cancer 88(10), 2239-2245 (2000).

- Indicates that VEGF expression is an important predictor for both liver metastasis and poor prognosis in ductal pancreatic adenocarcinoma.

12. N iedergethmann $M, H$ ildenbrand $R$, Wostbrock $B$ et al.: $H$ igh expression of vascular endothelial growth factor predicts early recurrence and poor prognosis after curative resection for ductal adenocarcinoma of the pancreas. Pancreas 25(2), 122-129 (2002).

- Indicates that VEG Fs may promote the distribution of metastases, leading to early cancer recurrence and poor outcome.

13. Itakura J, I shiwata T, Shen B, Kornmann M , Korc $\mathrm{M}$ : C oncomitant over-expression of vascular endothelial growth factor and its receptors in pancreatic cancer. Int. J. Cancer 85(1), 27-34 (2000).

14. Karayiannakis AJ, Bolanaki $H$, Syrigos KN et al.: Serum vascular endothelial growth factor levels in pancreatic cancer patients correlate with advanced and metastatic disease and poor prognosis. Cancer Lett. 194(1) 119-124 (2003).

- Emphasizes the value of serum VEGF as a pancreatic cancer progression marker and the usage of its clinical determination.

15. Kobayashi A, Yamaguchi T, Ishihara T et al.: U sefulness of plasma vascular endothelial growth factor in the diagnosis of pancreatic carcinoma: differential diagnosis, tumor progression, and patient survival. Pancreas 31(1), 74-78 (2005).

16. Bellone G, N ovarino A, Chiappino I et al.: Circulating vascular endothelial growth factor and interferon- $\gamma$-inducible protein-10 levels in pancreatic during chemotherapy. Anticancer Res. 25(5), 3287-3291 (2005).

17. Li $X$, Feng $G S$, Zheng $C S$ et al.: Expression of plasma vascular endothelial growth factor in patients with hepatocellular carcinoma and effect of transcatheter arterial chemoembolization therapy on plasma vascular endothelial growth factor level. World J. Gastroenterol. 10(19), 2878-2882 (2004)

18. Poon RT, Lau C, Pang R et al.: H igh serum vascular endothelial growth factor levels predict poor prognosis after radiofrequency ablation of hepatocellular carcinoma: importance of tumor biomarker in ablative therapies. Ann. Surg. Oncol. 14(6), 1835-1845 (2007).

19. $M$ CM eekin D S, Sill M W, Benbrook D et al.: A Phase II trial of thalidomide in patients with refractory endometrial cancer and correlation with angiogenesis biomarkers: a gynecologic oncology group study. Gynecol. Oncol. 105(2), 508-516 (2007).

20. Drevs J, Zirrgiebel U,

Schmidt-G ersbach CIM et al.: Soluble markers for the assessment of biological activity with PTK 787/ZK 222584 (PTK/ZK), a vascular endothelial growth factor receptor (VEG FR) tyrosine kinase inhibitor in patients with advanced colorectal cancer from two Phase I trials. Ann. Oncol. 16, 558-565 (2005).

21. Krejci P, D vorakova $D, K$ rahulcova $E$ et al.: FGF-2 abnormalities in B cell chronic lymphocytic and chronic myeloid leukemias. Leukemia 15, 228-237 (2001).

22. Elshal M F, M CC oy JP: M ultiplex bead array assays: performance evaluation and comparison of sensitivity to ELISA. M ethods 38(4), 317-323 (2006).

23. Chen R, Pan S, Brentnall TA et al.: Proteomic profiling of pancreatic cancer for biomarker discovery. $\mathrm{M} \mathrm{ol}$. Cell. Proteomics 4(4), 523-533 (2005).

24. Liao $Q$, Zhao YP, Yang YC: C ombined detection of serum tumor markers for differential diagnosis of solid lesions located at the pancreatic head. H epatobiliary Pancreat. D is. Int. 6(6), 641-645 (2007).

25. M isek DE, Patwa TH, Lubman D M et al.: Early detection and biomarkers in pancreatic cancer. J. N atl Compr. Canc. N etw. 5(10), 1034-1041 (2007).

26. Pistol-Tanase C: Caveolin-1: a marker for pancreatic cancer diagnosis. Expert Rev. M ol. Diagn. 8(4), 395-404 (2008).

27. Fujioka S, Yoshida K, Yanagisawa S et al.: Angiogenesis in pancreatic carcinoma: thymidine phosphorylase expression in stromal cells and intratumoral microvessel density as independent predictors of overall and relapse-free survival. Cancer 92(7), 1788-1797 (2001).

28. Kuwahara K, Sasaki T, Kuwada Y et al.: Expressions of angiogenic factors in pancreatic ductal carcinoma: a correlative study with clinicopathologic parameters and patient survival. Pancreas 26(4), 344-349 (2003).

- Supports the correlation between a high expression of VEGF and FG F-2 and poor prognosis in pancreatic ductal carcinoma. 


\section{Soluble angiogenic markers in pancreatic cancer - RESEARCH ARTICLE}

29. Chung $G$, Yoon $H$, Zerkowski P et al.:

Vascular endothelial growth factor, FLT -1, and FLK-1 analysis in pancreatic cancer tissue microarray. Cancer 106(8), 1677-1684 (2005).

30. Cooper BC, Ritchie JM, Broghammer CL et al.: Preoperative serum vascular endothelial growth factor levels: significance in ovarian cancer. Clin. Cancer Res 8(10), 3193-3197 (2002).

31. Kurschat P, Eming $S, N$ ashan $D$ et al.: Early increase in serum levels of the angiogenesisinhibitor endostatin and of basic fibroblast growth factor in melanoma patients during disease progression. Br. J. D ermatol. 156(4), 653-658 (2007).

32. Denizot $Y, D$ eArmas $R$, Caire $F$ et al.: The $Q$ uantitative analysis of $b F G F$ and VEGF by ELISA in human meningiomas. M ediators Inflamm. 6, 36376 (2006).
33. Smolej L, Andrýs C, Krejsek J et al.: Basic fibroblast growth factor (bFGF) and vascular endothelial growth factor (VEGF) are elevated in peripheral blood plasma of patients with chronic lymphocytic leukemia and decrease after intensive fludarabinebased treatment. V nitr. Lek. 53(11), 1171-1176 (2007).

34. Dudek $A Z, M$ ahaseth $\mathrm{H}$ : C irculating angiogenic cytokines in patients with advanced non-small cell lung cancer: correlation with treatment response and survival. Cancer Invest. 23(3), 193-200 (2005).

35. Pavel ME, $\mathrm{H}$ assler $\mathrm{G}$, Baum $\mathrm{U}$ et al.: Circulating levels of angiogenic cytokines can predict tumour progression and prognosis in neuroendocrine carcinomas. Clin. Endocrinol. (0xf.) 62(4), 434-443 (2005).
36. Fredriksson S, H orecka J, Brustugun OT et al.: M ultiplexed proximity ligation assays to profile putative plasma biomarkers relevant to pancreatic and ovarian cancer. Clin. Chem. 54(3), 582-589 (2008).

37. Lash GE, Scaife PJ, Innes BA et al.: Comparison of three multiplex cytokine analysis systems: Luminex, SearchLight and FAST Q uant. J. Immunol. M ethods 309(1-2), 205-208 (2006).

38. Young SH, Antonini JM, Roberts JR et al.: Performance evaluation of cytometric bead assays for the measurement of lung cytokines in two rodent models. J. Immunol. M ethods 331(1-2), 59-68 (2008).

39. Saif M W: Anti-angiogenesis therapy in pancreatic carcinoma. JOP 7(2), 163-173 (2006). 